\title{
Determinants of high-risk human papillomavirus infection in anogenital warts
}

\author{
Goknur Ozaydin-Yavuz ${ }^{1}$, Serap Gunes Bilgili', Huseyin Guducuoglu², Ibrahim Halil Yavuz ${ }^{1}$, Sema Elibuyuk-Aksac', \\ Ayse Serap Karadag ${ }^{3}$ \\ ${ }^{1}$ Department of Dermatology, Faculty of Medicine, Van Yuzuncu Yil University, Van, Turkey \\ ²Department of Medical Microbiology, Faculty of Medicine, Van Yuzuncu Yil University, Van, Turkey \\ ${ }^{3}$ Department of Dermatology, Medeniyet University, Goztepe Research and Training Hospital, Istanbul, Turkey
}

Adv Dermatol Allergol 2019; XXXVI (1): 76-81 DOI: https://doi.org/10.5114/ada.2019.82915

\begin{abstract}
Introduction: Genital warts are benign epithelial tumours caused by human papilloma viruses (HPV), and are sexually transmitted. Genotyping of genital HPV bears great clinical significance in terms of treatment planning, follow-up, and prevention strategies.

Aim: To evaluate the distribution of high-risk HPV infection types in patients diagnosed with anogenital warts.

Material and methods: A total of 66 patients with anogenital warts were enrolled. Punch biopsy samples were obtained from the lesions of each patient. After nucleic acid purification and DNA extraction, the presence of HPV DNA was ascertained using the PCR method, followed by HPV DNA genotyping. The relationship between HPV type distribution and age, gender, clinical location, and number of sexual partners was investigated.

Results: Genotyping was performed and HPV genome was detected in 50 tissue samples (75.8\%). Low-risk genotypes predominated with a prevalence of $62.1 \%(42 / 66)$. The most prevalent genotypes were HPV- 6 (47\%), and HPV-11 (13.6\%). Other types detected included HPV-18 and HPV-3.

Conclusions: Genotyping of HPV provides significant clinical information regarding this family of viruses that play a role in the aetiology of a variety of genital cancers, as some of these malignancies are now considered preventable due to recent development of vaccines. We believe that our results may provide guidance on future vaccination programs in our country.
\end{abstract}

Key words: genital wart, human papilloma virus, anogenital wart.

\section{Introduction}

Genital warts are benign epithelial tumours caused by human papilloma viruses (HPV) and represent the most common type of sexually transmitted diseases worldwide [1, 2]. A high transition rate (65\%) and frequent recurrences are associated with decreased quality of life and increased economic burden [3, 4]. Major risk factors for genital warts include the number of partners, barrier contraceptive use, young age at the first sexual intercourse, circumcision, and male sexual behaviour. Although the sexual intercourse is the leading form of transmission, direct hand contact or indirect fomite transmission have also been reported [5]. Until now, although nearly 230 HPV genotypes have been identified, only 40 of these are associated with anogenital infections [6].
Genital HPV genotypes are divided into high- and low-risk groups in terms of their cancer-causing potential. The high-risk HPV genotypes include 16, 18, 31, 33, $35,39,45,51,52,56,58,59,68,73$ and 82, while, HPV- 6, $11,40,42,43,44,54,61,70,72,81,89$ are considered lowrisk genotypes $[6,7]$. In recent years, $20-50 \%$ of patients with genital warts have been found to harbour high-risk HPV genotypes. Also, epidemiological studies have indicated high rates of transmission from one partner to the other. Therefore, an individual carrying high-risk genotype of HPV affects not only that individual, but also his/ her partner(s) [5].

HPV genotyping bears clinical significance with respect to treatment planning, patient follow-up, and development of prevention strategies. In vitro cultures cannot be performed for HPV. Although HPV lesions can be readily diagnosed histopathologically, HPV genotyp-

Address for correspondence: Goknur Ozaydin-Yavuz MD, Department of Dermatology, Faculty of Medicine, Van Yuzuncu Yil University, 65100 Van, Turkey, phone: +90 5054753362, e-mail: goknuroz1@gmail.com

Received: 3.12.2017, accepted: 28.12.2017. 
ing is pathologically unfeasible and molecular tests are required [8].

\section{Aim}

In this regard, we aimed to determine the age, gender, previous treatments, marital status, and number of partners in a group of patients with genital warts from Eastern Turkey and to perform HPV genotyping using the polymerase chain reaction (PCR) method.

\section{Material and methods}

This study was undertaken with the participation of patients who were clinically diagnosed with genital warts at the Dermatology Outpatient Unit. This study was approved by the Clinical Ethics and Research Committee of the Yuzuncu Yıl University, Faculty of Medicine (Date: 13.03.2014, Number: 11). Age, gender, occupation, number of sexual partners, the duration of the disease, previous treatments, and number of recurrences were recorded into patient follow-up forms and a detailed examination was performed for each patient. Serological tests including HIV, anti HCV, anti HBs, HBs Ag, Treponema pallidum haemagglutination (TPHA) test, and venereal disease research laboratory (VDRL) test were performed. After local intradermal anaesthesia, tissue samples from the lesions were obtained for PCR testing using punch biopsy technique, followed by electro-cauterization. All patients provided written informed consent after they were provided with information on the nature and purpose of the study procedures. Tissue samples obtained from each patient were placed in a tube containing $2 \mathrm{ml}$ of phosphate-buffered saline and were kept at $-80^{\circ} \mathrm{C}$ until the time of PCR analysis and genotyping. Following nucleic acid purification and extraction, the presence of HPV DNA was ascertained using the nested PCR method. In samples positive for HPV genome, HPV DNA genotyping was performed.

\section{DNA extraction}

The majority of molecular assays cannot be performed on a cell that is structurally intact: the DNA content must be first separated from the remainder of the cell. For the SuPerLy study, a commercially available kit was used for this procedure. The reasons for this were to be as consistent and reliable as possible and to use approved systems that could also be used in clinical laboratory settings. The QIAamp MinElute Media Kit (Qiagen, Hilden, Germany) was chosen because it is suitable for use with liquid media containing nucleic acids. The QIAamp MinElute Media Kit is designed to ensure that there is no sample-to-sample cross contamination and ensure safe handling of potentially infectious samples. There are four steps of the procedure including: lyse, bind, wash, and elute. The samples are lysed at high temperature denaturing conditions using proteinase $\mathrm{K}$ and two lysis buffers. The buffers increase lysis efficiency and ensure inactivation of RNases. Binding of the nucleic acids to the QIAamp MinElute column membrane is facilitated by adding ethanol to the lysates followed by high-speed centrifugation. Two wash steps are required to remove any contaminants from the membrane. Finally, the pure nucleic acids are eluted into the buffer. One of the most simple and inexpensive ways of DNA extraction involves proteinase K. Contaminating proteins, including a number of nucleases, are degraded by the addition of proteinase K. This method was applied to some of the residual clinical material.

\section{Amplification and melting}

The following conditions have been optimized on the "Rotor-Gene Q" using the amplification reagents contained in the kit. The instrument should be switched on at least 20 min before the start of the reaction. Before starting, the AMP dye solution should be brought to room temperature and mixed thoroughly in a vortex mixer. Once opened, the AMP dye solution should be kept at $+2 /+8^{\circ} \mathrm{C}$ and used within a month, otherwise it needs be frozen. It should not be thawed and re-frozen more than twice. The reagents must be mixed thoroughly in a vortex mixer before use and then centrifuged briefly. Work should be done quickly in ice or on refrigerated blocks. One should bear in mind that each session must include at least one negative amplification control WATER, the HPV sign ${ }^{\circledR}$ pos ctrl and the specificity control h-DNA ctrl.

\section{Pyrosequencing method}

Samples with appropriate bands were diluted by adding $27 \mu \mathrm{l}$ of PCR product to $13 \mu \mathrm{l}$ of sterile water. The PyroMark Q96 Vacuum Prep Workstation was prepared with the appropriate solutions (1: 10 diluted wash buffer, $70 \%$ ethanol, water (deionised and autoclaved), denaturation buffer) and the vacuum prep tool head was rinsed thoroughly with water for $20 \mathrm{~s}$. Details of the reaction and samples were input into the PyroMark CpG Software. Pyrosequencing PCR was performed using a biotin labelled primer. PCR products were immobilised by adding $1.75 \mu$ l of streptavidin sepharose bead suspension and $38.25 \mu \mathrm{l}$ of PyroMark Binding Buffer per reaction. The mixes were then shaken on a shaking hot plate for at least $5 \mathrm{~min}\left(1400 \mathrm{rpm}, 22^{\circ} \mathrm{C}\right)$. Sequencing primers were made up by diluting (per reaction) $1.5 \mu \mathrm{l}$ of $10 \mu \mathrm{M}$ sequencing primer with $43.5 \mu$ l of PyroMark Annealing Buffer. Forty-five $\mu$ l of sequencing primer mix was dispensed into each well of a pyrosequencing (PSQ) reaction plate and the plate was placed into the correct compartment of the vacuum workstation. The PCR product/sepharose bead mix was removed from the hotplate and placed into the correct position on the vacuum workstation. Within $30 \mathrm{~s}$ of cessation of shaking, the vacuum was applied 
and the sepharose beads with immobilised PCR products were captured by slowly lowering the vacuum prep tool into the PCR plate. The vacuum prep tool was then placed into each of the following solution trays (in order) for $5 \mathrm{~s}$ each; $70 \%$ v/v ethanol solution, PyroMark Denaturation Solution, 10\% v/v PyroMark Washing Buffer. The prep tool was held vertically and any residual fluid was aspirated. The prep tool was lowered into the PSQ reaction plate containing sequencing primer and the vacuum switch was closed whilst hovering above the solution and then agitated to release the captured PCR products. The PSQ plate containing beads and sequencing primer was heated at $80^{\circ} \mathrm{C}$ for $2 \mathrm{~min}$, then cooled to room temperature. The PyroMark Gold Q96 Reagent kit contained lyophilised enzyme and substrate pellets as well as dNTP mixes. Enzyme and substrate were reconstituted with the volume of water specified on the container 10 min prior to use. The PSQ96 Reagent Cartridge was filled using the volumes specified by the pyrosequencing software and the wells specified. The cartridge and reaction plate were placed into the PyroMark Q96 ID Instrument and the run was started. At the end of the run, the data were analysed automatically and a program was produced for each pyrosequencing reaction.

\section{Analysis of the results and identification of the genotype}

Apart from the results obtained with PyroMark Q24 sw. Usually the colour of at least one of the four wells in which each clinical sample has been sequenced is blue (Good quality) or yellow (Check quality). All the clinical specimens were analysed, and so were the amplification and sequencing controls with the IdentiFire sw independently from the results obtained with PyroMark Q24 sw. The file HPV library contained in the HPV sign ${ }^{\circledR}$ Q24 CD was used, checking that its review matches that indicated on the "HPV sign ${ }^{\circledR}$ Q24 complete" kit being used.

Table 1. HPV genotypes in patients with anogenital warts

\begin{tabular}{lcccccc}
\hline HPV genotype & \multicolumn{2}{c}{ Female } & \multicolumn{2}{c}{ Male } & \multicolumn{2}{c}{ Total } \\
\cline { 2 - 7 } & $n$ & $\%$ & $n$ & $\%$ & $n$ & $\%$ \\
\hline HPV 6 & 5 & 7.6 & 26 & 39.4 & 31 & 47.0 \\
\hline HPV 11 & 3 & 4.5 & 6 & 9.1 & 9 & 13.6 \\
\hline HPV 18 & 1 & 1.5 & 0 & 0 & 1 & 1.5 \\
\hline HPV 3 & 1 & 1.5 & 0 & 0 & 1 & 1.5 \\
\hline $\begin{array}{l}\text { Unclassified } \\
\text { infection }\end{array}$ & 3 & 4.5 & 5 & 7.6 & 8 & 12.1 \\
\hline $\begin{array}{l}\text { Cases of HPV } \\
\text { negative }\end{array}$ & 0 & 0 & 16 & 24.2 & 16 & 24.2 \\
\hline \begin{tabular}{l} 
Total \\
\hline
\end{tabular} & 13 & 19.7 & 53 & 80.3 & 66 & 100 \\
\hline
\end{tabular}

The QIAGEN kit enables the detection of $37 \mathrm{HPV}$ genotypes (18 high-risk genotypes and 19 low-risk genotypes), as follows: high-risk HPV genotypes, 16, 18, 26, 31, $33,35,39,45,51,52,53,56,58,59,66,68,73$, and 82; and low-risk HPV genotypes 1, 2, 3, 4, 5, 6, 8, 11, 13, 30, 34, 40, 42, 43, 44, 67, 69, 70, and 71 .

\section{Statistical analysis}

Data were analysed using Statistical Package for the Social Sciences (SPSS) 18.0 statistics software. For the comparison of parametric variables, $t$-test was used, while intergroup comparisons for categorical variables were performed with the $\chi^{2}$ test. A $p$-value less than 0.05 was considered statistically significant.

\section{Results}

A total of 66 patients between 18 and 55 years of age (mean age of $31 \pm 8.76$ years) who had a disease duration (mean) of $15.97 \pm 25.8$ months were included. There were 53 (80.3\%) male and 13 (19.7\%) female participants. Table 1 shows the results of HPV genotyping. Although HPV genotyping was performed in all patients, HPV could not be detected in 16 (24.2\%) male patients. Unclassified infections that tested positive for HPV DNA by PCR but did not hybridize a specific HPV type occurred in $12.1 \%$ of genital warts.

The most common site of lesions was the pubic area followed by penile localization ( $n=21,31.8 \%$ ) (Figure 1). Table 2 shows the age, disease duration range for patients with anogenital warts, the distribution of the anatomic location of the lesions and demographic characteristics of the patients. Of the overall subjects, $21.2 \%(n=14)$ were self-employed and $16.7 \%(n=11)$ were military personnel. Forty-one (62.1\%) patients were married, while 25 (37.9\%) were single. Genital warts were present in the partner of $9(13.6 \%)$ patients, while in $57(86.4 \%)$ individuals, no genital warts could be detected in the partner. Thirty-four (51.5\%) individuals had no previous treatment versus 32 (48.5\%) with a previous history of treatment. Cryotherapy was the most common form of ( $n=42$, $63.6 \%$ ) previous therapy, followed in the decreasing of frequency by a combination of cryotherapy and electrocautery ( $n=13,19.7 \%)$, podophyllin $(n=6,9.1 \%)$, electrocauterization $(n=3,4.5 \%)$, and imiquimod $(n=1,2 \%)$.

A recurrence had occurred in $44(66.7 \%)$ patients. The recurrence rate was not significantly associated with the gender and number of partners ( $p=0.27$ and 0.456 , respectively). However, the treatments significantly differed according to gender $(p=0.033)$. Accordingly, women received podophyllin and cryotherapy most frequently, as compared to significantly higher rates of cryotherapy among male patients. Again, male patients reported a significantly higher number of sexual partners than female patients $(p=0.012)$. Female patients were found to have a significantly higher occurrence of HPV genotypes 3 and 18 ( $p=$ 0.010 ). Again, the male gender was associated with a significantly increased likelihood of having a negative HPV result 
Table 2. Demographic characteristics of patients with anogenital warts $(n=66)$

\begin{tabular}{|c|c|c|}
\hline Variable & $N$ & $\%$ \\
\hline \multicolumn{3}{|l|}{ Number of partners of study subjects: } \\
\hline Single & 30 & 45.5 \\
\hline Multiple & 34 & 51.5 \\
\hline No partner & 2 & 3.0 \\
\hline \multicolumn{3}{|l|}{$\begin{array}{l}\text { Presence of genital warts in partners of } \\
\text { patients: }\end{array}$} \\
\hline Yes & 9 & 13.6 \\
\hline No & 57 & 86.4 \\
\hline \multicolumn{3}{|c|}{$\begin{array}{l}\text { Positive serological test results in patients with anogenital } \\
\text { warts: }\end{array}$} \\
\hline Anti-HBs & 18 & 27.3 \\
\hline HBsAg & 0 & 0 \\
\hline TPHA & 0 & 0 \\
\hline VDRL & 0 & 0 \\
\hline Anti-HCV & 0 & 0 \\
\hline Anti-HIV & 0 & 0 \\
\hline Circumcision (present in all male patients) & 53 & 80.3 \\
\hline \multicolumn{3}{|l|}{ Anatomical location of genital warts: } \\
\hline Pubic area, Penile shaft & 21 & 31.8 \\
\hline Pubic area & 16 & 24.2 \\
\hline Pubic area, Penile shaft, Scrotum & 10 & 15.2 \\
\hline Perianal & 8 & 12.1 \\
\hline Labia major and minor & 3 & 4.5 \\
\hline Penile shaft & 3 & 4.5 \\
\hline Scrotum & 2 & 3.0 \\
\hline Introitus & 2 & 3.0 \\
\hline Labia major, Pubic area & 1 & 1.5 \\
\hline
\end{tabular}

( $p=0.023$ ). Marital status also was significantly associated with the number of partners, married individuals being significantly more likely to have a single partner $(p=0.027)$.

\section{Discussion}

In patients participating in our study, HPV genotypes $6,11,18$, and 3 were identified. Although most genital warts arise from HPV type 6 or 11 infections, high-risk genotypes were particularly more frequently found in patients with genital warts due to HPV 16, 18, 31, and 33 $[7,9]$. The role of high-risk HPV genotypes in the development of cervical cancer as well as anal, vulvar, and penile

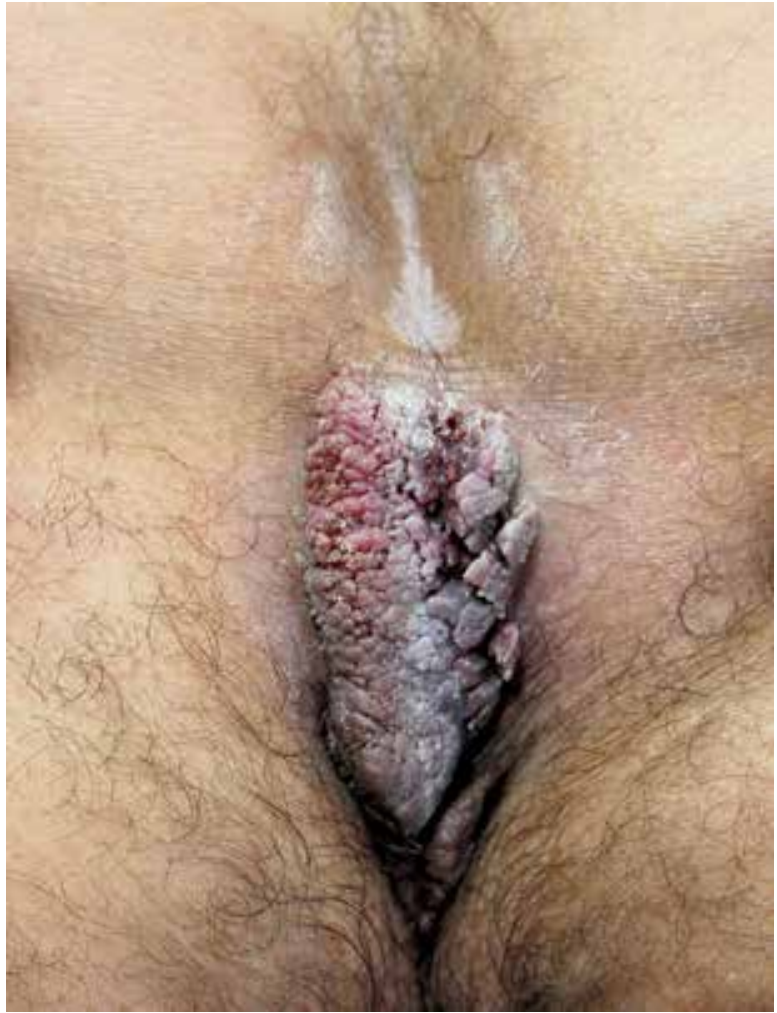

Figure 1. Genital wart of the anal margin

cancers is now clearly evident. Therefore, genotyping of HPV infections bears clinical significance and has been subject to extensive research. In a study by Jamshidi et al. from southern Iran, samples obtained from a total of 100 women with a mean age of 26 years were tested for HPV genotyping with PCR method. Genotyping could be performed in 73 (73\%), while it was not possible in the remaining 27 (27\%) patients. In that study, only HPV 6 and 11 genotypes were detected at a frequency of $49 \%$ and $67 \%$, respectively [6]. In our study, the type of HPV could not be identified in $12.1 \%$ of the patients $(n=8)$, which may be due to unclassifiable HPV genotypes.

Anic et al. was able to perform HPV genotyping with PCR in only 90 (80.4\%) of 112 male patients with genital warts. The most common non-oncogenic types were type 6 (43.8\%) and type 11 (10.7\%). In that study, multiple HPV types were present in $45 \%$ of the patients, and $47 \%$ of the individuals had non-oncogenic HPV, while 6 (5.4\%) only had oncogenic types. Of the oncogenic genotypes, HPV 16 (9.8\%) and HPV 52 (6.2\%) were the most frequent [10]. In a study by Park et al., genotyping with PCR was performed among 150 male patients with histopathologically-documented genital warts. Fifteen patients were excluded due to negative HPV PCR. Low-risk genotypes (HPV-6 and 11) were identified in 128 patients, while 31 were found to have high-risk genotypes (HPV-16, 33, 18, and 68). A single-virus infection was present in $87 \mathrm{pa}$ - 
tients versus multiple HPV infection in 41 patients. The most common genotype was HPV-6. A total of 13 patients were uncircumcised and had increased frequency of oncogenic HPV genotypes than circumcised men [1].

In a study by Ingles et al., genital warts were histopathologically confirmed in 228 of 377 patients with external genital type lesions. The incidence of high and low risk of HPV genotypes in those with external genital lesions was $15.6 \%$ and $73.2 \%$, respectively. The most commonly identified HPV genotypes were HPV-6 and 11 [11]. In a multi-centre study by Chang et al. involving 18 centres and a total of 1005 patients, after initial histopathological confirmation, HPV genotyping was performed in 891 subjects. The distribution of genotypes of HPV across male and female subjects was comparable. Low- and high-risk HPV genotypes were detected in $78.1 \%$ and $11.6 \%$, respectively, the most common genotypes being HPV-6 and 11 [12].

Similar to other reports, HPV was present in $75.8 \%$ of the genital warts in our study. When patients with negative HPV were excluded, these figures rose to $62 \%$ and $18 \%$. One patient was found to have high-risk HPV (HPV-18). In eight individuals, genotyping could not be performed although presence of HPV was detected using PCR. In this regard, it is interesting to note that genotyping could be generally performed in other studies, while there were unclassifiable HPV forms in the current study. This may be due to the unclassifiable types of HPV. Again in contrast with some other studies reporting multiple HPV genotypes in certain lesions, we had no such cases with multiple HPV genotypes. This may be due to methodological differences. In the study by Ingles et al., oncogenic HPV genotypes were found at a much higher incidence than in our study [11]. We believe that this might be related with the fact that all male patients in our study were circumcised, which may be a protective factor against oncogenic strains. In the study by Campion et al., lesions harbouring oncogenic HPV were found in $32 \%$ of the partners of male patients with genital warts [13]. In our study, $13.6 \%$ of the partners were found to have genital warts, although genotyping was not performed in the partners.

In another study by de Lima Rocha et al., penile samples were obtained from male partners $(n=43)$ of women with cervical HPV infection and at least one genotype of HPV was found in these samples, with high-risk HPV frequency of 33\% [14]. These studies suggest that genital warts may also pose a risk for the patient. Most of the studies of HPV genotyping involved female patients, with relative scarcity of genotyping in male subjects. This is mainly due to the occurrence of cervical cancer in women requiring additional attention for HPV genotyping. In our study, most of the patients attending our unit with genital warts were male, female patients representing 19.7\% of the study population. On the other hand, only female patients had the high-risk genotypes (HPV-18 and 3).
Although skin samples obtained from genital warts are important, other non-skin material has also been used for investigations. For instance, Nielson et al. obtained semen samples in addition to genital warts [15]. Johnson et al. performed HPV genotyping in urinary samples from 3123 patients [16]. In studies involving female patients, mainly cervical smear samples have been utilized. Studies involving direct genotyping from the genital warts are scarce in number.

In our country, only one previous study looked at HPV genotypes in genital warts. In the study by Serdaroglu et al., HPV type 6 and 11 were found in $88 \%$ and $11.9 \%$ of the cases, respectively [17]. Similarly, in our study HPV-6 (47\%) and 11 (13.6\%) were the most frequently observed genotypes. Contrary to this previous report, one patient had HPV-3 and another had HPV-18.

Therapeutic approaches for the treatment of genital warts include podophyllin, trichloroacetic acid, cryotherapy, electrocautery, imiquimod, carbon dioxide laser, and surgery [18]. In our study patients, the most frequent forms of therapy were cryotherapy (63.8\%) and electrocautery (19.7\%). Despite best treatment, a recurrence ranging between $40 \%$ and $90 \%$ has been reported for genital warts [5]. Accordingly, the recurrence rate in our study was $66.7 \%$.

\section{Conclusions}

The first-generation HPV vaccines, that is quadrivalent HPV type 6/11/16/18 vaccine and bivalent HPV type 16/18 vaccine were licensed in 2006 and 2007, respectively. Recently a second-generation nine-valent HPV type $6 / 11 / 16 / 18 / 31 / 33 / 45 / 52 / 58$ vaccine has been licensed. It is expected that with the vaccine the incidence of genital warts will decrease within a period of decades. Vaccination programs against HPV are becoming increasingly important when one considers the associated economic burden, high recurrence, stigmatization and embarrassment as well as reduced quality of life in this condition. Thus, there are scarce studies involving HPV genotyping in our country, and we believe that this study may shed some light on the future vaccination programs. Inclusion of HPV genotypes 6 and 11 in addition to oncogenic HPV genotypes in vaccination programs may lead to a decrease in the occurrence of genital warts, with an accompanying decline in the economic and social burden.

\section{Acknowledgments}

This work was supported by the Research Fund of the Yuzuncu Yil University. Project Number: 2014-HIZ-TF185.

\section{Conflict of interest}

The authors declare no conflict of interest. 


\section{References}

1. Park SJ, Seo J, Ha SH, Jung GW. Prevalence and determinants of high-risk human papillomavirus infection in male genital warts. Korean J Urol 2014; 55: 207-12.

2. Dempsey AF, Koutsky LA. National burden of genital warts: a first step in defining the problem. Sex Transm Dis 2008; 35: 361-2.

3. Alam M, Stiller M. Direct medical costs for surgical and medical treatment of condylomata acuminata. Arch Dermatol 2001; 137: 337-41.

4. Wiley D, Masongsong E. Human papillomavirus: the burden of infection. Obstet Gynecol Surv 2006; 61 (6 Suppl 1): S3-14.

5. Dupin N. Genital warts. Clin Dermatol 2004; 22: 481-6.

6. Jamshidi M, Shekari M, Nejatizadeh AA, et al. The impact of human papillomavirus (HPV) types 6, 11 in women with genital warts. Arch Gynecol Obstet 2012; 286: 1261-7.

7. Munoz N, Bosch FX, de Sanjosé S, et al. Epidemiologic classification of human papillomavirus types associated with cervical cancer. N Eng J Med 2003; 348: 518-27.

8. Chen X, Li L, Lai Y, et al. Characteristics of human papilloma viruses infection in men with genital warts in Shanghai. Oncotarget 2016; 7: 53903-10.

9. Garland SM, Steben M, Sings HL, et al. Natural history of genital warts: analysis of the placebo arm of 2 randomized phase III trials of a quadrivalent human papillomavirus (types 6, 11, 16 , and 18) vaccine. J Infect Dis 2009; 199: 805-14.

10. Anic GM, Lee JH, Stockwell H, et al. Incidence and human papillomavirus (HPV) type distribution of genital warts in a multinational cohort of men: the HPV in men study. I Infect Dis 2011; 204: 1886-92.

11. Ingles DJ, Pierce Campbell CM, Messina JA, et al. Humanpapillomavirus virus (HPV) genotype- and age-specific analyses of externalgenital lesions among men in the HPV Infection in Men (HIM) sStudy. J Infect Dis 2015; 211: 1060-7.

12. Chang L, Ci P, Shi J, et al. Distribution of genital wart human papillomavirus genotypes in China: a multi-center study. J Med Virol 2013; 85: 1765-74.

13. Campion MJ, Singer A, Clarkson PK, McCance DJ. Increased risk of cervical neoplasia in consorts of men with penile condylomata acuminata. Lancet 1985; 1: 943-6.

14. de Lima Rocha MG, Faria FL, Gonçalves L, et al. Prevalence of DNA-HPV in male sexual partners of HPV-infected women and concordance of viral types in infected couples. PLoS One 2012; 7: e40988.

15. Nielson CM, Flores R, Harris RB, et al. Human papillomavirus prevalence and type distribution in male anogenital sites and semen. Cancer Epidemiol Biomarkers Prev 2007; 16: 1107-14.

16. Johnson AM, Mercer CH, Beddows S, et al. Epidemiology of, and behavioural risk factors for, sexually transmitted human papillomavirus infection in men and women in Britain. Sex Transm Infect 2012; 88: 212-7.

17. Serdaroglu S, Akkurt M, Kuşkucu M, et al. The determination of HPV types in anogenital warts and research of it's relationship with recurrence. Dermatoz 2010; 1: 173-6.

18. Aubin F, Pretet JL, Jacquard AC, et al. Human papillomavirus genotype distribution in external acuminata condylomata: a large French national study (EDiTH IV) Clin Infect Dis 2008; 47: 610-5. 